la modification de la concentration des autres constituants du sérum qui est obtenue simultanément et dans les mêmes proportions avait pu coopérer à ce résultat.

Nous avons constaté, de plus, que lors de la dilution de la crème, d'une part, avec du lait écrémé acidifié, d'autre part, avec de l'eau, la teneur en matière grasse du babeurre était plus élevée dans le dernier mélange.

Traduction: R. N. Göransson.

\title{
LE SÉRUM DE CASÉINERIE ET QUELQUES SOUS-PRODUITS DE L'INDUSTRIE LAITIÈRE : LEURS PROPRIÉTÉS ET LEUR VALEUR ALIMENTAIRE
}

\author{
par le Dr Paul ROSSI \\ Direnteur Régional des Services Vétérinaires de Saône-et-Loire
}

(Fin).

Les biochimistes américains, MAC-Collum et DAvis, OsBorne' et Mendel, ont montré la spécifité de certains amino-acides dans l'entretien ou la croissance. « La lysine apparaît comme un aliment de croissance indispensable aux jeunes; une ration sans lysine, mais complète par ailleurs, ne peut permettre le développement; l'adulte peut mieux s'en passer, mais réclame du tryptophane pour son entretien " (31). Pour qu'il y ait croissance par la lysine, il faut d'abord qu'il y ait entretien par le tryptophane; le développement demande de la cystine (OsBorne et Mender), de l'arginine et l'histidine (Hopkins et Ackroyd). "La proline et le glycocolle peuvent être éliminés sans que leur absence se fasse sentir " (32). "D'autres, alanine, valine, sont nécessaires quoique indifférentes. Les recherches récentes d'ABDERHALDEN tendent à mettre la leucine parmi les acides-aminés indispensables ". (33).

La lactalbumine, étant prise comme étalon, c'est elle qui paraît la plus complète, pour le moindre taux, de toutes les albumines connues (31). C'est une "protéine positive " (RAMon Banco) qui, par les proportions optimum de sa lysine, son tryptophane, sa leucine, son arginine, son histidine, etc. (16), apportera l'azote de qualité définie, nécessaire à la formation des muscles et des autres tissus et à la couverture de la dépense azotée. Or la croissance et le développement du porc se poursuivent pendant toute sa vie économique. L'augmentation de poids est déterminée, non seulement par l'accumulation de graisse, mais aussi par la formation de chair et autres tissus. Aucun aliment ne saurait mieux convenir que le lacto-sérum.

Il ne suffit pas de donner de l'azote ; il faut, avant toute chose, qu'il puisse être utilisé dans l'organisme. L'utilisation digestive de la lactalbumine n'a pas été sensiblement modifiée par le chauffage à $63^{\circ}$, au cours des manipulations de la préparation du sérum. Terroine et 
d'autres (34) ont observé que les taux d'utilisation du lait de vache, par le porc, sont exactement les mêmes que pour l'enfant; la spécifité d'origine n'entraîne pas une différence de la digestibilité, mais la fixation d'azote par le jeune animal à croissance impétueuse ne laisse que $50 \%$ des matières protéiques qu'il apporte; " ce gaspillage, si considérable en apparence, est cependant bien intérieur à celui des autres albuminoïdes, dont le taux d'utilisation ne dépasse pas $20 \%$ et est souvent inférieur à $16 \%$ " (32). "La lactalbumine ne commence à devenirinsuffisante que pour un taux égal ou inférieur à 4,5 de la ration (31).,

$\mathrm{La}$ méthode des équivalents fourragers nous indique que la ration d'entretien d'un pore, à l'engrais, est assurée par une unité nutritive environ. Or, une unité nutritive est représentée par 12 litres de sérum (35). En Charente-Inférieure, la ration normale d'un pore est de 20 litres de sérum; le pore y trouvera, à peu près, l'azote suffisant pour assurer la couverture de tous les besoins si, avec LAPICQUE, l'on admet qu'un gramme d'azote par kilog correspond aux besoins azotés (36); encore cette évaluation serait-elle trop élevée. $D$ 'après les expériences de Zelier, de Dessarezet Bterry (34), le besoin en azote est minimum quand la dépense énergétique est assurée par les hydrates de carbone, condition réalisée avec le lactose du sérum. L'administration de sérum, sans ration complémentaire, ne pourrait cependant qu'être fâcheuse pour les raisons que nous allons énumérer, maintenant. La teneur en eau, $92 \%$ environ, est trop considérable. La digestion et l'assimilation ne peuvent se faire favorablement sans la présence d'un " caput mortuum n ou tout au moins d'aliments, présentani un certain volume et un certain poids. Les limites de la possibilité d'assimilation seraient trop vite dépassées (a).

La valeur alimentaire d'un aliment ne se déduit pas seulement de son analyse chimique, mais aussi de son analyse biologique. Le sérum est un aliment vivant, qui, en plus des "facteurs minimaux " précédents, apporte des vitamines.

Les travaux innombrables, qui ont suivi les expériences mémorables de Hopkins et de FUNCK, montrèrent qu'íl existe dans les aliments " des substances de nature chimique, encore indéterminée, quoique serrée de près, de rôle energétique minime, mais d'une importance fonctionnelle essentielle " (38). Ce sont les " vitamines " ou "facteurs accessoires » dont le nombre et la complexité croissent au fur et à mesure que leur étude s'approfondit. Les unes sont liposolubles, résistent à de hautes temperatures, aux alcalis, à la dessiccation; ce sont les vitamines proprement dites :

Facteur de croissanee

(a) D'après le Professeur Porcher (communication personnelle), en Argentine, les porcs ne recevraient que du sérum de fromagerie, 40 à 50 litres par jour et plus, sans autre complément de ration. 


\section{Facteur antirachitique}

Facteur de reproduction.

Les autres sont hydrosolubles :

Facteur antibéribérique

Facteur antiscorbutique.

"La richesse du lait et du sérum, en vitamines, est proportionnelle a celle des aliments, car la glande mammaire n'a pas la faculté d'en faire la synthèse ; elle les élimine, comme le rein excrète l'urée ou le chlorure de sodium " (39).

La Vitamine A, appelée aussi liposoluble A ou facteur A, a une composition chimique inconnue; elle n'est détruite que, lentement, aux hautes températures. Par contre, l'action combinée de la chaleur et de l'oxygène la détruit rapidement; elle résiste mal aux rayons ultraviolets. "Son absence dans l'alimentation entraîne des troubles dans la nutrition qui se manifestent constamment par l'arrêt de la croissance et dans la majorité des cas par des lésions des paupières et de la cornée »*

$\mathrm{Au}$ cours de la centrifugation, le facteur $\mathrm{A}$ suit la matière grasse du lait et ne se rencontre plus, par la suite, dans le sérum, qu'en quantité infime ou nulle, toujours insuffisante pour mettre l'organisme à l'abri dos troubles qui, pour MouriquaND, ne peuvent être évités qu'en ajoutant, àu ne ration dépourvue de liposoluble, du beurre au taux de $5 \%$.

Le facteur antirachitique, liposoluble aussi, serait absent du sérum pour des raisons identiques à celles du facteur $\mathrm{A}$.

Le facteur B, soluble dans l'eau, l'alcool faible, l'huile d'olives, résiste aux acides (28), est détruit par les alcalis et lentemert par la chaleur à $120^{\circ}(40)$. Il est nécessaire à l'entretien et à la croissance. L'expérimentation montre fréquemment qu'un régime dépourvu de facteur B, même s'il est riche en. A, ne permet pas la croissance. Son absense entraîne surtout des troubles nerveux et cutanés. Elle donnerait le béribéri.

La Vitamine B existe, dans le sérum, en quantité assez importante, bien que, d'après SrmonNET, son émonctoire naturel soit plutôt le rein que la mamelle. « De nature azotée, elle est énergiquement adsorbée par le lactose dont on ne peut la séparer que par des cristallisations successives " (41). Elle joue un rôle principal dans le fonctionnement de l'économie, dans l'augmentation du poids du corps et dans la digestion (42). Sous son influence, si la proportion de certains ferments digestifs (trypsine et stéapsine) ne semble pas modifiée, celle d'autres ferments, l'amylase pancréatique surtout, serait notablement augmentée; or, la nourriture du porc est essentiellement riche en hydrates de carbone, sucre, amidon etc., dont la digestion est assurée par l'action de l'amylase ; un régime abondant en facteur B, comme le régime de sérum de lait, ne peut qu'être favorable à la meilleure utilisation de la ration. Il faut un certain équi- 
libre entre la quantité de facteur B et la quantité d'hydrates de carbone, présents dans un régime. Il semble dangereux d'introduire une proportion très forte d'hydrates de carbone, si la proportion de facteur B n'est pas en même temps augmentée.

La substance " antiscorbutique " est de nature " chimique complètement inconnue, soluble dans l'eau et l'alcool, dialysable, plus sensible aux alcalis qu'aux acides, elle résiste peu à l'action de la chaleur et surtout à celle de l'oxygène (43). " Cette vitamine qui n'existe pas dans la caséine et le beurre, mais dans Je mélange lactose-sels (puisque le lactose pur n'est pas antiscorbutique, il faut admettre qu'elle est en dissolution dans l'eau du lait) (44), se retrouve-t-elle dans le sérum de caséinerie ? Elle en a disparu au cours des manipulations. La cuisson à $63^{\circ}$, pendant 30 minutes au contact de l'air, a facilité l'oxydation, que le cuivre des cuves à caséine a encore accrue, comme il résulte des travaux de Hess et WeINSTOCK (45), confirmés par VAN LeERSTM, [d'Amsterdam (46) et par SUPPLEe (47). Les bacilles lactiques du sérum sont incapables d'enrichir celui-ci, en facteur antiscorbutique (WoLlmans, 48).

La Vitamine C est nécessaire à l'entretien; elle a une action sur la nutrition des os, des épithéliums vasculaires et sur l'hématopoièse. A-t-elle sur la croissance une action spécifique comparable à celle des facteurs A et B ? MourTQUAND a montré que son absence ne modifiait pas, d'une façon appréciable, la croissance des jeunes sujets dans la période première du scorbut qui précède l'apparition des signes osseux caractéristiques. "La substance antiscorbutique est plutôt un facteur d'équilibre. Son rôle à ce point de vue paraît moindre que celui des autres facteurs non identifiés A et B; sels, et surtout amino-acides " (28).

Les Vitamines agissent à doses faibles (38): Mais on s'imagine cependant volontiers que des traces d'aliments suffisent à empêcher les manifestations aiguës des carences. "Il y a plus qu'une condition de présence nécessaire, il y a aussi une nécessité de quantité, même de quantité considérable » (49).

Le porc, recevant avec le régime du sérum, une nourriture déficitaire en vitamines, tout au moins en facteur liposoluble $\mathrm{A}$, en facteur antirachitique et en facteur antiscorbutique, est-il sujet aux troubles avitaminosiques ? La susceptibilité du porc aux carences alimentaires a fait l'objet de nombreuses études expérimentales, surtout à l'étranger. De l'avis presque unanime, le jeune pore, très sensible au défaut de certains facteurs accessoires, se distingue des autres anjmaux domestiques et surtout des ruminants, qui montrent une résistance marquée, que l'on attribue parfois à l'élaboration de vitamines par la flore microbienne, si riche et si variée de leurs réservoirs gastriques. Le sexe n'a aucune influence.

L'effet du défaut de vitamines n'est pas immédiat chez le porc, mais se fait sentir au bout de deux mois environ. La manifestation; la 
plus fréquente de la carence en facteurs accessoires du groupe liposoluble, est un arrêt du développement ou une prédisposition au rachitisme. Tout d'abord, l'état général devient mauvais, la croissance se ralentit, puis s'arrête définitivement (50).

Le béribéri pourrait apparaître sur le pore (HART MinLER) (5I); certains auteurs nient cette possibilité (FrEY) (52).

Un scorbut typique a été obtenu expérimentalement par GoLDING (53).

Les besoins du porc en vitamines des groupes $\mathrm{A}$ et $\mathrm{C}$ se sont montrés relativement bas, ear heureusement des réserves sont faites par le jeune, en lactation.

Sur les reproducteurs, en stabulation et nourris d'une façon trop monotone, deux effets nétastes des avitaminoses se font sentir :

Atrophie des organes génitaux et stérilité;

Pauvreté en vitamines du lait des mères.

Frey (52), se basant sur les études de quelques expérimentateurs, fait jouer aux vitamines un rôle important dans l'étiologie et la marche des maladies infectieuses. Il pense que chez le porc, l'absence de Vitamines peut a voir une influence dans l'étiologie des cas sporadiques de maladies infectieuses; surtout de celles dont la contagion n'est pas sûrement établie. Elle eréerait la prédis position, attendue par un microbe saprophyte pour devenit pathogène.

"Peu de pores meurent d'une carence, mais sous l'influence de celle-ci, nombreux peuvent être les malades, les retardataires et les rachitiques. Les déchets et les pertes sont assez considérables pour qu'il ne soit pas inutile d'attirer l'attention des nourrisseurs et plus spécialement de ceux utilisant certains sous-produits de laiterie, privés de crème " (54). "Les carences frustes " sont plus fréquentes chez le pore que les "carences totales" ou absolues. Dans la pratique, il est difficile de différencier ce qui, dans l'étiologie des accidents observés, revient en propre à chaque Vitamine, car leurs actions s'associent ou se chevauchent (55).

En raison de sa teneur en acide lactique et de sa richesse en bacilles lactiques, le sérum a été recommandé pour le traitement de certaines affections intestinales.

Après ce rapide exposé, pouvons-nous considérer le sérum comme le simple véhicule d'une ration complémentaire ou comme un simple exoitant de l'appétit? C'est pourtant l'opinion de DoRnic et Chollet qui éorivent : "Sa valeur alimentaire est, comme on le voit, très réduite ; on l'emploie surtout dans l'alimentation des pores. Il joue alors le rôle d'un a péritif ; ces animaux sont, en effet, très friands et cela permet de leur taire consommer de grosses quantités d'aliments de toutes sortes : Farine, tourteaux, résidus industriels divers" (1). C'est aussi l'avis d'André Gourn et ANDOdaRD, qui ajoutent : " La valeur nutritive du sérum est trop faible pour permettre de l'employer à hautes doses, 
quelque réduit que soit son prix. Mais son goût attire les porcs et permet de leur faire facilement a bsorber des aliments dont ils se soucient médiocrement s'ils sont simplement délayés dans l'eau " (56).

Nous ne partageons pas ces opinions et considérons le sérum comme un réel aliment, dont la valeur nutritive ne se déduit pas de sa seule teneur en protéine, car les autres éléments interviennent aussi. La croissance et le maintien de l'équilibre ne se résument pas essentiellement en un phénomène protéique, ainsi qu'il résultait de la vieille doctrine de BUNGE : Les graisses et les hydrates de carbone jouent un rôle, aussi important que les albuminoïdes, si toutefois on fait appel à un minimum de ces dernières (57). Pour éviter des déchets onéreux et pour maintenir par des mélanges avec d'autres aliments, tourteaux, grains, certaines relations, certains rapports entre les divers éléments, l'on ne peut employer, seul, le sérum, et l'on doit distribuer généralement un complément de ration.

Les quantités de sérum, produnt par les laiteries coopératives de la Charente-Inférieure (Enquête laitière 1925), s'élèvent à plus de 160 millions de litres:

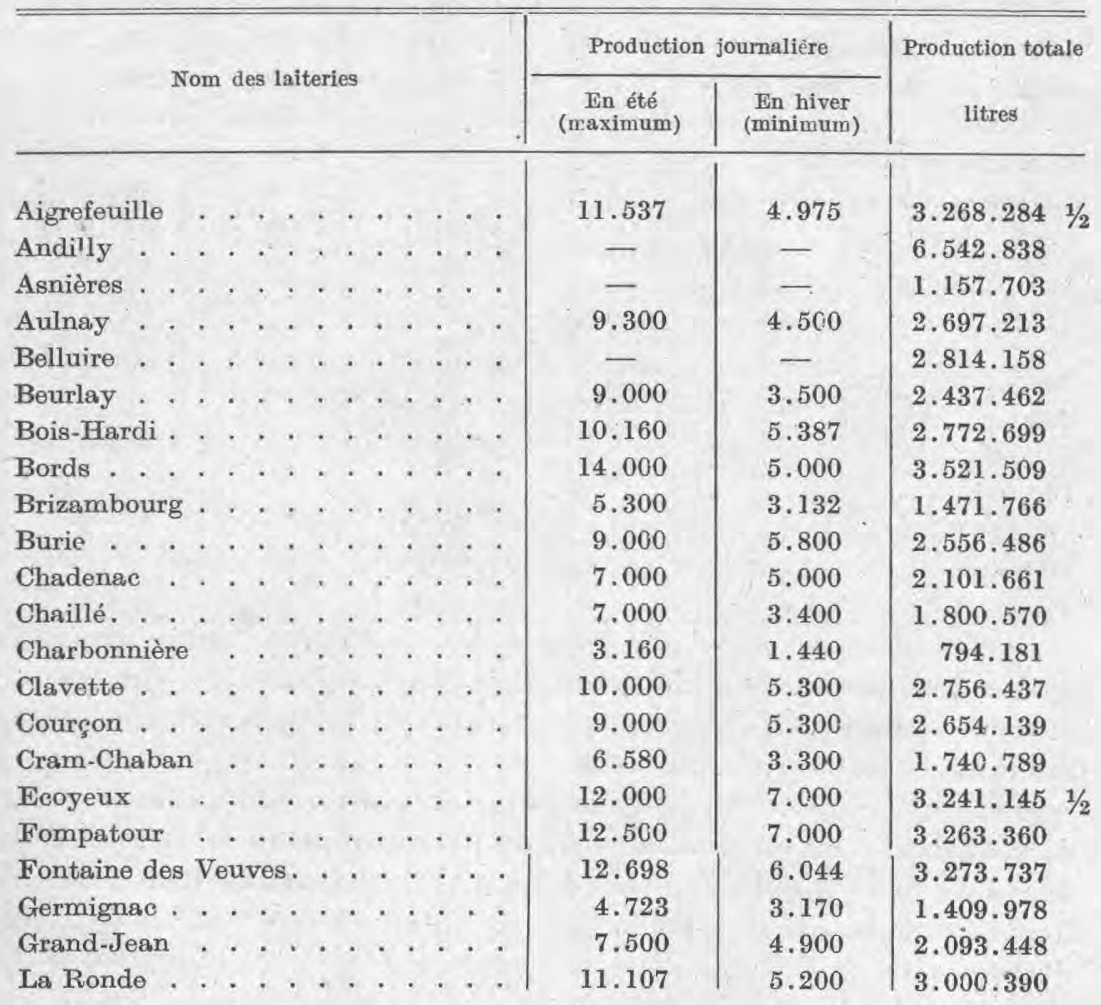




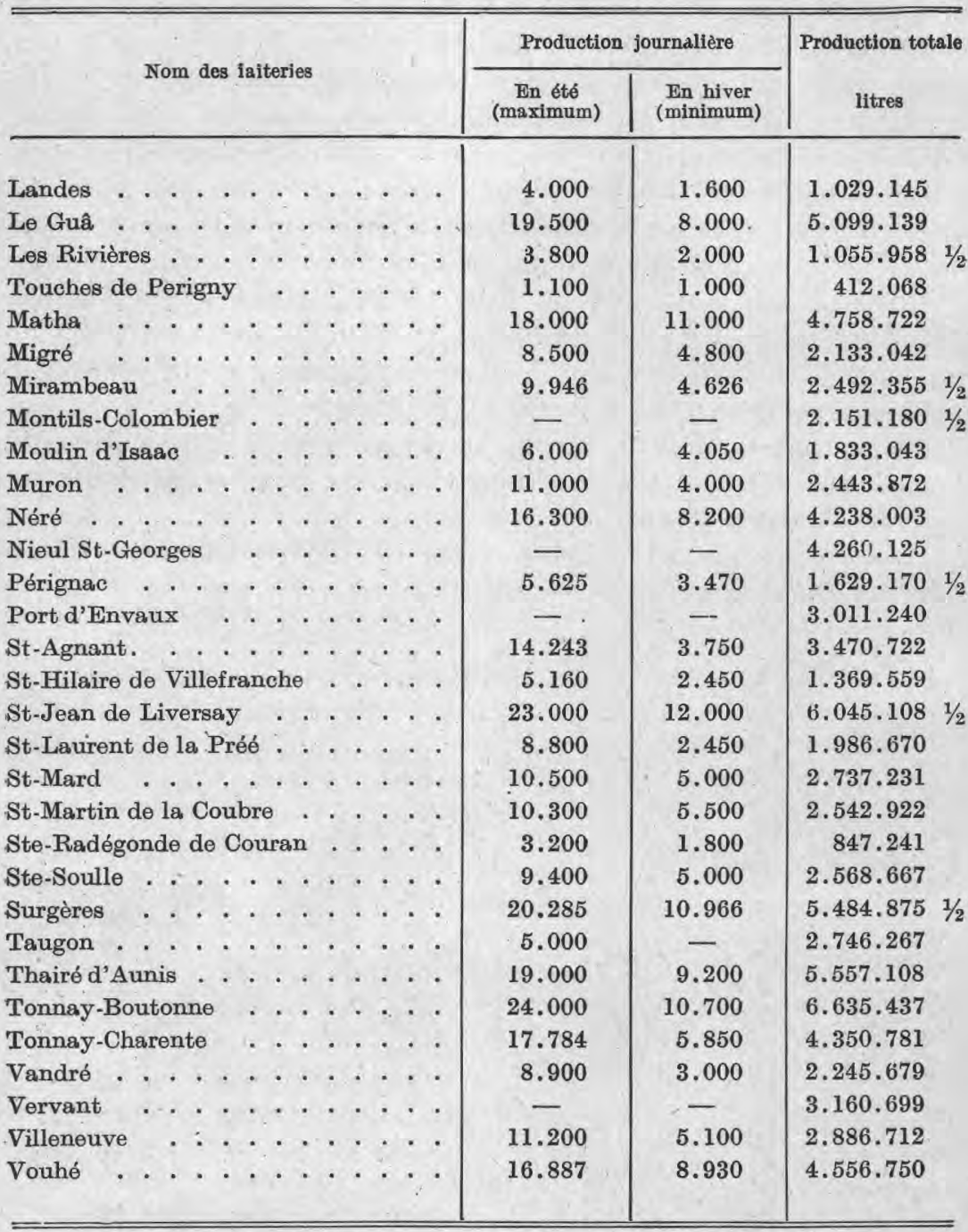

Un sous-produit du lait mériterait d'être signalé, qui est encore inconnu, malgré tout l'intérêt qu'il présente : C'est le sérum concentré, résidu de la préparation du lactose (a).

Par concentration dans le vide à une température inférieure au point de coagulation de la lactalbumine, on obtient d'abord le "sérum condensé ", liquide très fluide à chaud, mais visqueux comme de la crème à froid, d'une densité de 1.260 à $54^{\circ} \mathrm{C}$. et d'un volume égal au $1 / 12^{\circ} \mathrm{du}$

(a) L'industrie du lactose est rudimentaire en France. 
volume primitif. On en retire la majeure partie du lactose. Il reste alors un résidu, (58).

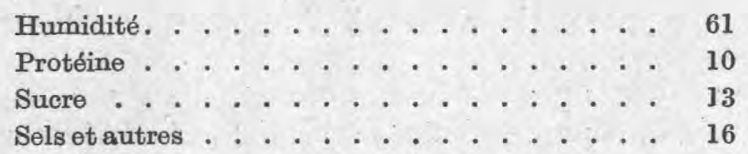

qui peut être desséché facilement sur des rouleaux ou mieux encore concentré dans le vide. Le "Whey Paste " obtenu,

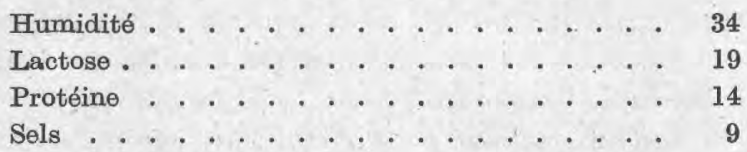

difficile à manipuler à cause de sa consistance gommeuse, doit malgré sa conservation parfaite, être consommé directement (58). Aussi, préfère-t-on le mélanger à des résidus de distillerie ou de brasserie et obtenir, selon le degré de dessiccation auquel on s'arrête, le "Lactose Feed " ou le "Whey food ". (58).

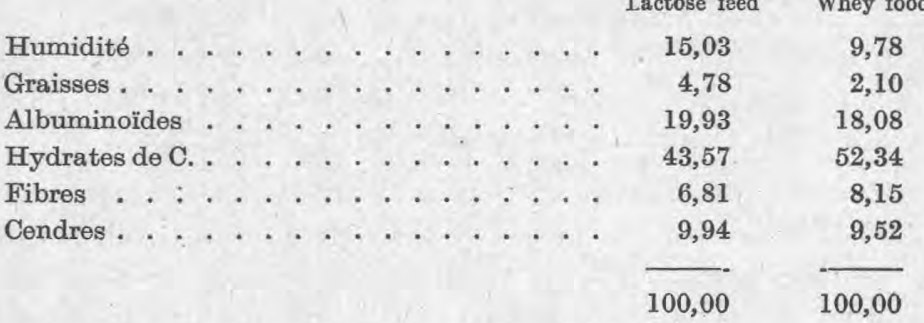

La quantité de protéine digestible s'élève à $15 \%$.

Golding (59) (communication personn elle)étudie actuellement un système de rouleaux dessécheurs, donnant un rendement plus intéressant.

RIEDel (60), après concentration selon un procédé particulier (chaudière dans laquelle le sérum est projeté sur des ailes tournantes), ajoute du son au sérum concentré. Son "Molkenkleie » (son au petit lait) est un produit pulvérulent, d'odeur agréable, d'une saveur analogue à celle du pain légèrement sucré. La composition est la suivante :

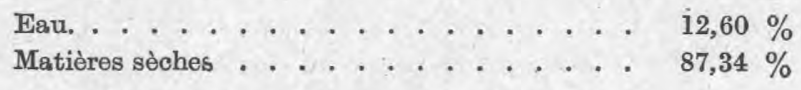

La matière sèche peut se décomposer ainsi :

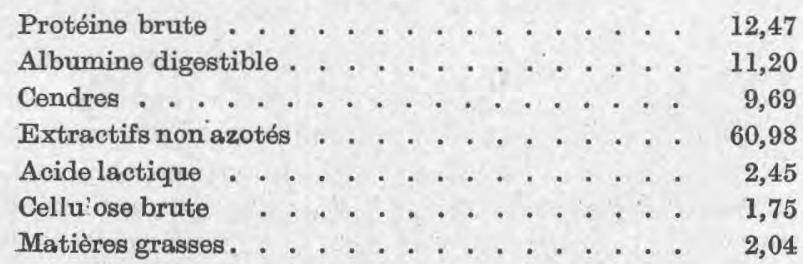


L'avantage du sérum concentré serait de pouvoir réserver en été, époque de la grande production laitière, un aliment pour l'hiver, saison où le lait est plus rare. Le gaspillage énorme actuel diminuerait considérablement.

L'influence, sur la croissance et l'entretien des porcs qui gagnent plus de poids que les témoins et sont de meilleure qualité, semble être três favorable. L'économie, réalisée par l'addition de $10 \%$ de ces dérivés à la ration normale, serait très marquée (61).

Lait écrémé. - Le lait écrémé n'est mentionné, ici, que pour mémoire, car depuis l'ère de la caséine, sa consommation a presque disparu en Charente-Inférieure. Dans un avenir très proche, toutes les laiteries extrairont la caséine. La composition du lait écrémé est la suivante :

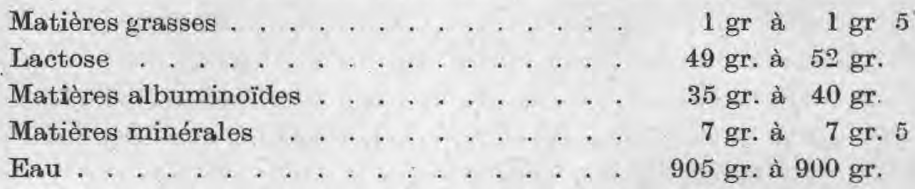

Sa richesse en azote trop élevée, sa relation nutritive (c'est-à-dire le rapport de la matière azotée aux matières non azotées) trop étroite, entraînent quelques sérieuses difficultés pour son emploi : Les porcs viennent difficilement. Le lard reste mou.

Babeurre. - Le babeurre, lait de beurre ou lait battu (a), liquide résiduel de la fabrication du beurre, s'écoule dans la baratte au moment de la soudure des globules gras. C'est un liquide blanc, opaque, homogène, d'odeur et de saveur agréables et de réaction légèrement acide. Au repos, il se dispose en deux couches : l'inférieure, homogène, blanche, composée de grumeaux de caséine coagulée; la supérieure, jaunâtre, n'est que du lacto-sérum. Malgré les variations peu négligeables, dues aux procédés de fabrication du beurre (crème douce, crème acide, addition d'eau pour faciliter la prise du beurre, etc.), la composition moyenne se rapproche sensiblement de celle du lait écrémé. Les auteurs n'indique pas les mêmes proportions de composants :

\begin{tabular}{|c|c|c|c|c|c|c|}
\hline & & Modigliani & Péhu (63) & Dorlencourt & Kufferath (64) & Fleischmann \\
\hline Matières grasses . & . . & 3,5 & 6,6 & 5,2 & 5,6 & 56 \\
\hline Caséine ..... & . . & 37,8 & 32,4 & 37 & " & 33 \\
\hline Albumine ..... & . . & b &,$\infty$ & w & n & 2 \\
\hline... & . & 39,1 & 32 & 36 & 40 & 40 \\
\hline Cendres...... & . . & 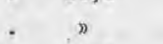 & 》 & $n$ & » & 7 \\
\hline Extrait sec total. & . . & 89,9 & 》 & 84 & 87,6 & $n$ \\
\hline " $\quad$ dégraiesé & é. & $n$ & ” & n & 82 & ” \\
\hline Acide lactique . . & . . & 3,2 & 4,54 & 5,94 & ” & $n$ \\
\hline
\end{tabular}

(a) En diététique humaine, le terme de babeurre englobe une variété de produits différents les uns des autres. MARFAN désigne sous ce nom le lait écrémé acide. 
L'acidité du babeurre, due surtout' à la fermentation lactique, dépend de la température ambiante, comme pour le sérum. Le babeurre contient de nombreux bacilles lactiques. La richesse en bacilles lactiques purs augmente quand la crème a été pasteurisée et ensemencée pour faciliter la maturation.

La matière grasse, qui ne devrait jamais dépasser 3 à $4 \%$ avee des manipulations bien conduites, atteint fréquemment 7 à $10 \%$. La teneur en lactalbumine est plus grande que celle du lait. La caséine du babeurre, moins abondante, ne précipiterait pas par le labferment, mais subirait plus énergiquement que la caséine du lait, l'action de la trypsine (65). Des modifications chimiques de cette caséine découlent des changements dans l'état des sels insolubles du lait: Les phosphates seraient transformés en sels organiques solubles. Le babeurre, plus riche en lécithines que le lait de vache, aurait, d'après DoHnal (65), une assez forte proportion de pseudo-nucléine $(1,1 \%)$.

Les laiteries coopératives disposent quotidiennement de quantités élevées de babeurre qu'elles mélangent au sérum ou abandonnent avec les eaux résiduaires. 100 litres de crème fournissent environ 56 litres de babeurre.

Non dilué, sa valeur alimentaire est exactement identique à celle du lait écrémé, ainsi que l'ont établi de nombreuses expériences, notamment celles de Morrisson, dans l'Ohio, l'Arkansas, l'Indiana et le Wisconsin. Il fournirait 350 calories. Trop riche en protéine et en eau, il doit être mélangé à des céréales ou des farines et consommé en quantités limitées (EvVARD (66). L'optimum paraît être environune ou 3 parties de babeurre pour une partie de grains ; au-dessus, le porc utilise mal ou peu économiquement la protéine.

A l'Etranger, l'industrie, par des procédés utilisés pour le lait, a réussi à condenser et même à dessécher le babeurre comme l'a fait HUNZIKER (67).

Cette poudre, d'une conservation parfaite à l'abri de l'air et à basse tempárature, correspond à 10 volumes de babeurre naturel.

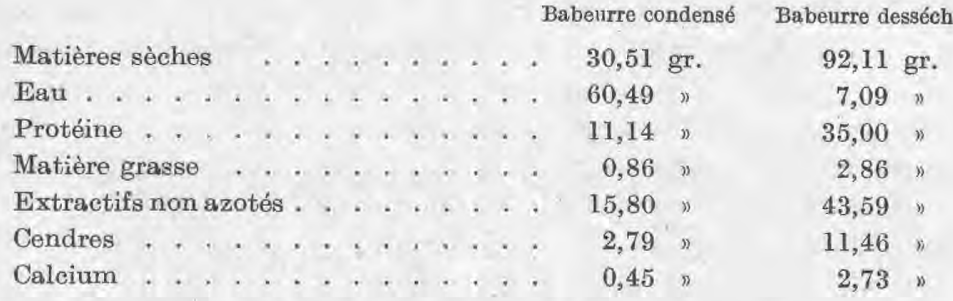

Ces babourres, condensés ou desséchés, renferment environ $3 \%$ d'acide lactique (69). Les Vitamines A et B persistent, l'antiscorbutique C a été détruite. En Hollande, à la suite des travaux de GraAmbon, le babeurre condensé est additionné de sucre et de farine, puis vendu en 
boîtes de fer blanc pour les enifants. Ces produits, surtout l' "Eatall ", ont donné à Dunn et Evvard (70) de splendides résultats sur le porc. Ils sont encore inconnus en France, où les succédanés du babeurre n'ont été introduits que pour l'alimentation des enfants et notamment par Blechman (Société de Pédiâtrie).

De nombreux pédiâtres, NoBécourt, Marfan, préconisent le babeurre naturel dans les affections digestives de l'enfance. IESN l'emploie avec succès dans le Prurigo Strophulus, dans la maladie de Barlow. Marcel LabBé le signale pour le traitement des dyspepsies et des entérocolites (Traité des régimes alimentaires).

En Charente-Inférieure, la réputation du babeurre n'est pas toujours excellente, car maintes épidémies de fièvre aphteuse du porc peuvent lui être attribuées. Aussi, dès l'apparition de cette épizootie dans le rayon d'une laiterie, arrête-t-on la livraison de ce sous-prcduit. (Le sérum porté à $63^{\circ}$ est stérile du point de vue aphteux). La production du babeurre desséche serait, d'après EckLes et GaLLickson, très économique et pourrait aisément concurrencer d'autres aliments.

Nous voyons donc que l'industrie laitière peut fournir des sousproduits d'une haute valeur alimentaire, d'ailleurs parfaitement utilisés en Charente-Inférieure. Il serait souhaitable cependant d'éviter le gaspillage et d'envisager, là où le porc ne peut le conscmmer, la transformation du sérum en sérum concentré.

D'autre part, le régime du sérum ne va pas sans à-coups souvent sérieux: Des études physiologiques consciencieuses permettraient de mieux connaître la valeur alimentaire du sérum. Les engraisseurs de porcs, directement intéressés, pourraient largement aider les laboratoires, les stations de recherches, en leur fournissant les sujets d'étude et aussi l'aide pécuniaire.

\section{BIBLIOGRAPHIE}

[1] Dornic et Chollet. Lait, beurre et dérivés. (Baillère, Paris, 1926).

[2] Rice Frank et Markuey Autor. Rapport entre l'acidité naturelle du lait, sa composition et ses caractères physiques. (Journ. of Dairy Science, sept. 1924, p. 468 à 483$)$.

[3] Monvorsin. Le Leit, $3^{\ominus}$ édition. (Vigot, Paris, 1925).

[4] Cité par HaRdrNG. Whey problem. A solution. (Ministry of Agrieulture and fisheries research; monograph, $\left.\mathrm{n}^{\circ} 5,1925\right)$.

[5] Berry. Journ. Agr. Scienc. Vol, XIII, Fasc. 2, avril 1923.

[6] Porcher et Chevaliter. La répartition des matières salines dans le lait, leurs relations physiques et chimiques avec les antres principes du lait. (Le Lait, 1923, p. 97 et suivantes).

[7] Gyoray. Sur la répartition du calcium et du phosphore inorganique dans le lait. (Biochemische Journal, nos 1 et 2, octobre 1923). 
[8] Muller. Dosage titrimétrique der phosphates dans le lait : Emploi pour l'appréciation du lait. (Mitteillungen aus dem Gebiete des Lebensmittel Untersuchung, mars 1922).

[9] Dornic et Datre. Revue Générale du Lait, T. VIII, page 385.

[10] Bordas et RACzkowsky. Comptes rendus Académie des Sciences, 1913.

[11] Porcher. Revue Générale du Lait, $5^{\mathrm{e}}$ année, page 193.

[12] Pietrae. Sur les protéides du lacto-sérum. Leur séparation par la méthode àl'acétone. (Comptes rendus Académie des Sciences, 14 janvier 1924).

[13] Deniges. Thèse de pharmacie, Paris 1892.

[14] Bleyer et O. Kallmann. Contribution à la connaissance de quelques composants encore peu étudiés du lait. (Bioch. Zeitsch. T. CLIII, No 3, 1924, p. 459 et suiv.).

[15] Porcher. Le lait au point de vue physico-chimique. Sa constitution colloïdale. ( $V I I^{\mathrm{e}}$ Congrès international de Laiterie, Paris, 1926, p. 185).

[16] Luning et Herzig. Différenciation et dosage de la laçtalbumine dans un mélange de deux substances. (Zeitsch. für Untersuch. der Nahrung und. Genussmittel, 15 février 1921).

[17] SImonnet. La valeur alimentaire du lait. (Recueil de Médecine vétérinaire, 15 nov. 1920 , p. 535).

[18] Plimmer. The constitution of the proteins.

[19] Mouriquand. Les Vitamines et les nouvelles conceptions alimentaires. (Avenir Médical, février 1927, p. 47).

[20] OsBorne et MendeL. Journal Bioch. 1916).

[21] Miller. Journ, Biol. Chem., 1923, p. 61.

[22] SPATtel et TANzer. Ueber Kalk und Phosphorsaüre Bedarf und Fütterung beim, Schwein (Zeitsch für Schweinzucht, Fasc 10, p. 143, 1922).

[23] OrR et Husband. Scottish Journal Of Agriculture, Vol. 5, p. 244, 1922.

[24] Crichton et Mac Gowan. On the effect of deficiency ot iron in the diet of pigs. (The Bioch. Journal 1923, Vol. XVII, p. 204.)

[25] Laulanté. Elémente de physiologio

[26] Deighton. Basal métabolism of growing pigs. (Proceedings of the Royal. Society, Série B, Vol. XCV, p. 340, 1923.)

[27] Terrone et Mile Mende. De l'influence de l'adjonction d'aliments ternaires. au lait sur la grandeur de la rétention azotée au cours de la croissance. (Compte rendu Ac. des Sciences, 1923.)

[28] Mouriquand, Mrohel et Barre. Ration de croissance. (Journal de médecine. de Lyon, $n^{\circ}$ 99, 29 fév. 1924, p. 96.)

[29] Terrotne. Conférence à l'Institut Solvay, fóvr. 1920.

[30] Srmonnet. Alimentation du bétail bovin et contrôle laitier. (Compte-rendu du. Congrès de l'alimentation du bétail, 1925, p. 21 à 65.)

[31] STMONnet. Idées nouvelles sur l'alimentation. (Revue de Zootechnie, 1921, p. 129 et suiv.).

[32] Hopkins et ACKROYD. Feedings experiments with deficiencies in the aminoacids supply, arginine and histidine as possible précursors of purins. (Biol. Journ., T. X, 1926, p. 551).

33 ] ABDERHALDEN. Weitere Beiträge für Kenntnis von organischen Nahrungstoffen mit spezifischer Wirkung. (Arch. f. die ges. Physiol., T. CXCV. p. $199-226,1926$.) 
[34] Terroine et Spindler. Influence des procédés de pasteurisation sur la digestibilité des albuminoïdes et minéraux du Lait. (Le Lait, mars 1925, p. 241 et suiv.).

[35] A. Leroy. L'alimentation par la méthode des équivalents fourragers. Le Lait, 1923, p. 352.)

[36] LaPICQue. Compte rendu Société Biologie, 17 mars 1894.

[37] DesGrez et Biery. Equilibre azoté et hydrates de carbone dans la ration alimentaire. (Compte rendu Académie des sciences, T. CLXXI, 1920, p. 1393 à 1396.)

[38] Roger. Nouveau traité de Médecine. (Masson 1924, fascicule 7, p. 1.)

[39] Lesne et Vagliano. Les vitamines du lait. (Le Lait, 1925, p. 955.)

[40] Slanetz E. Influence du chauffage à l'autoclave sur la teneur en vitamines du lait. (Journ. of Dairy Sciences, Vol. VI, n⿳0 3, mai 1923.)

[41] Mme Randorn et Srmonnet. Bulletin de la Société d'Hygiène alimentaire, 1926, $\mathrm{n}^{\circ} 5$, p. 215.

[42] Sogen Tokusige et Tokeo Odoka. De l'influence de la Vitamine B sur la digestion. (Journ. Biochimistry. Tokio 1922, T. I, p, 465.)

[43] Lesné. Vitamines du lait. (Le Lait, 1925, p. 955.)

[44] Lesné et MHe Dubrevil. Du pouvoir antiscorbutique des différents éléments du lait. (Bulletin Société Pédiatrie, 1923, p. 29.)

[45] A. F. Hess et Weinstock. Catalytic Action of Minute accounts of Copper in destruction of antiscorbutic Vitamin in Milk. (Journ. Am. Méd. Assoc., 12 mars 1924, T. LXXXII, no 12, p. 952.)

[46] Van Lefroum. Pasteurisation et teneur en vitamines. (VII ${ }^{\mathrm{e}}$ Congrès international du Lait, Paris 1926.)

[47] Supplee. Le cuivre dans le lait. Son influence sur les Vitamines. (13th Annual Report of inter. Assoc. of Dairy and Milk inspec., 18-20 oct. 1924, p. 37 à 51.)

[48] Wolmann. Sur le rôle des microorganismes dans la production des vitamines. (Compte rendu Soc. Biol. no 31, 1921, p. 801.)

[49] Goduewsky. Carence partielle et préscorbut. (Presse Médicale, 27 août 1927, p. 682).

[50] Golding, Drummond, Zinva. Relation of the fat soluble factor to rickets and growth in pigs. (Biol. Journ. Vol. XVIII, p. 879-881, 1924 et Vol. XVI, p. 392 à 401,1922 .)

[51] Hart Mrluer et MaC Collum. Journ Bioch. Chemistry, Vol. XXV, 1926.

[52] Frey. Vitaminen, Avitaminosen und Infektions Kranheiten bei Tieren. (Schweiz Arch. für Tierheilkunde, 1926, p. 164.)

[53] Golding, Drummond, Zilva et Coward. Relation of the fat soluble factor to rickets and growth in pigs. (Bioch. Journ. Vol. XV, 1922, p. 427.)

[54] Rossi Paul. Contribution à l'étude des carences alimentaires. (Revue Gén. de Méd. Vét. Fév. 1927, p. 65 et suiv.)

[55] Rossi Paul. Les Vitamines dans la nutrition du pore. (Revue de Zootechnie, $\mathrm{n}^{\circ} 8$, août 1927 , p. 110 et suiv.)

[56] André Gounn et Andouard. Vie d̀ la campagne, 15 oct. 1920.

[57] Amar. Croissance et interaction alimentaire. (Comptes rendus Ac. des Sciences, 1926, T. II, no 8 , p. 544.) 
[58] Golding et Moriss. Whey research factory at Haslington. Pig feeding experiments with lactose residues. (Journ. of. Ministry of Agriculture, T. XXXII, no 10, Janv. 1926, p. 911 à 918.)

[59] Golding. Communication personnelle.

[60] Riedex. Molkenwerwertung. (Molkerei Zeitung, 1923, n 100, p. 1841 à 1842.)

[61] Oka et Crichton. The food value of dried whey solids. (Scottish Journ. of Agr.,

Vol. VI, 1923, p. 63 à 67.)

[62] Cité par Chasseray. Le babeurre, aliment, médicament. (Rev. Pratique des maladies des Pays chauds, T. III, n० 2, von. 1923.)

[63] Doriencourt et Penu. Société de Pédiatrie, 5 juin 1920.

[64] Kufferath. A propos des laits battus. (Annales, de Gembloux, $\mathrm{n}^{\text {os }} 8$ et 12 , août et décembre 1923.)

[65] Dhonal. Action du babeurre sur la sécrétion gastrique. (Monatssch. für Kinderheillunde, T. XXVII, No 1, p. 58 à 64, oct. 1924.)

[66] JoHn Evvard. A Little Milk Work Wonders in pig feeding. (Iowa experiment station, Bul. 17.)

[67] Hunziker. Utilisation du babeurre sous forme de babeurre condensé et desséché. (Journ, of Dairy Science, janv. 1923, Vol. VI, no 1, p. 1 à 12.)

[68] Eckles et Gallickson. Condensed and powdered buttermilk for dairy calves. (Journ. Of Dairy Science, mai 1924, Vol. VII, n 3, p. 213 à 221.)

[69] Hunziker. Oondensed Milk and Mille powder, $4^{\mathrm{e}}$ édition 1926. La Grange Illinois, p. 522 \& 530 .

[70] John Evvard et Russelu Dunn. Dried Butter milk for pigs. (Iowa Experiment station, Bul. 58.)

[71] John Evvard et Russell Dunn. Milk by products in their bit in pork making. (Iowa Agr. Experiment station.)

\title{
LA MÉTHODE SYNTHÉTIQUE DANS L'ÉTUDE DU LAIT. LE LAIT AU POINT DE VUE COLLOIDAL. RECHERCHES SUR LE MÉCANISME DE L'ACTION DE LA PRÉSURE
}

\author{
par Ch. PORCHER,
}

Docteur ès sciences physiques

(Suite)

\section{CHAPITRE VIII.}

\section{LES APPORTS SALINS DANS LE LAIT}

Dans la première partie de ce travail, nous avons vu quels étaient les sels qu'il fallait employer pour séparer la caséine de la globuline et de l'albumine. Rappelons qu'en général, ce sont toujours des quantités massives auxquelles on a recours.

Nous n'avons fait aucune allusion au retentissement que l'addition de ces sels pourrait avoir sur l'emprésurage, puisque c'est d'un tout autre objet qu'il s'agissait. Etant donné, ainsi que nous le verrons plus 